\title{
Research on the Development of Micro-Tourism Market in Changsha City Under the Background of the Internet Era
}

\author{
Peng Chen ${ }^{1} \&$ Jia-Wei Tang ${ }^{1}$ \\ ${ }^{1}$ Central South University of Forestry and Technology, Changsha, China \\ Correspondence: Peng Chen, Central South University of Forestry and Technology, Changsha 410000, Hunan \\ Province, China.
}

Received: October 10, 2019

Accepted: November 4, 2019

Online Published: November 6, 2019

doi:10.20849/ajsss.v4i4.665

URL: https://doi.org/10.20849/ajsss.v4i4.665

\begin{abstract}
With the advent of the 21 st century, China has entered the era of the Internet, and the tourism industry has continued to develop. The demand for domestic tourism and national leisure consciousness has been continuously improved. As the transformation of tourism forms has gradually shifted to leisure, experience and diversification, micro-tourism springs up. In 2011, micro-tourism was officially put on the agenda as an emerging form of tourism. After that, it became popular and developed rapidly. Large numbers of micro-tourism products dominated by 1-2 days of hot spring tour, ancient town tour, island tour, country tour, and suburb tour were launched online instantly. This paper first expounds the background, formation, development and characteristics of micro-tourism and then interprets the current development of micro-tourism from online survey data, finding that the development of micro-tourism market is good, but the related facilities and systems are comparatively lagging behind. Finally, the paper analyzes the micro-tourism resources and development conditions of Changsha city, finding that the foundation and market platform for developing micro-tourism in Changsha is sound. Given the actual development, the paper then puts forward development strategy for micro-tourism of Changsha City under the background of the Internet era, which may provide reference for other cities with similar conditions to develop micro-tourism.
\end{abstract}

Keywords: internet era, micro-tourism, micro-tourism market, Changsha City

\section{Introduction}

The 21 century is known as the information age, Internet age, technological age as well as age of big data. As Tencent officially launched its free application WeChat which provides instant messaging services for smart terminals in 2011, China has entered the micro age, and the society starts to move toward "short, flat and fast" development stage. In the micro age where the word "micro" is ubiquitous, there are a full range of new words in the form of "micro- $X$ " such as micro-friends, micro-wish, micro-films, micro-marketing, and micro-house. At the moment, micro life is popular and micro concepts are far-reaching. Meanwhile, the development of Internet and technology has also enabled tourism to form a huge sensing and network system, linking tourist attractions and urban public facilities into the network. Besides, with more transparent information, advanced transportation, and increasing short holidays, the concept of "pan-tourism" and "no tourist attractions" are emerging. Merely with leisure mood and instant decision to leave, one can go travelling at once. And the form of "micro-tourism" has become available in people's tourism activities. In recent years, the market of micro-tourism has increased rapidly. Compared with long-distance travelling, the form of micro-tourism is more simple. One can go travelling with a backpack. And the travel time and ways of travelling become more arbitrary. This kind of travel mode, featuring less preparation, short journey, simple procedure, and instant decision, has gradually become people's preferred choice.

Imbued with randomness, micro-tourism which covers short time and distance is a kind of travel decided by one's free mind. It enables people to temporarily "liberate" themselves from the busy schedule, relax their body and mind, and to avoid the tedious preparation of long-distance travel. In doing so, one can truly appreciate the nature, which constitutes the greatest charm of micro-tourism. In order to get an in-depth understanding of the development of micro tourism, this paper, utilizes online survey data and the method of comprehensive text analysis, analyzes the current situation, prospects and strategies of micro tourism against the backdrop of the internet, and puts forward corresponding countermeasures for the development of micro tourism in Changsha 
city in light of the development environment and conditions of micro tourism in Changsha city, and of the results of data analysis.

\section{Research Summary}

\subsection{On Micro-Tourism}

\subsubsection{The Beginning of "Micro" - Origin and Background of Micro-Tourism}

"Micro-tourism" is not the miniature version of conventional tourism products, but a new understanding of the idea of tourism, a new exploration of tourism modes and a new discovery of tourism objects. As an emerging tourism industry, micro-tourism has developed rapidly in just a few years, mainly by the following reasons: (1) the advent of the Internet era. The Internet has spawned a form of convenient interaction such as WeChat and Weibo. QQ, Fetion and mail systems that develop with the Internet technology have also assisted the development of micro tourism. For some local government agencies, Weibo has become an auxiliary tool for strengthening tourism management (Sun, 2013). In 2015, China's mobile phone users reached 668 million (Note 1), where the popularity of mobile Internet and WiFi provides giant wings for information exchange of micro tourism. Internet becomes an important way for potential tourists to exchange tourism information, make tourism decisions, travel and to publish their experiences. The emergence of various online travel Apps enables people to master first-hand information at anytime and anywhere. As the age of Internet is the micro age as well as the age of big data, people use media to spread new experiences during their trip, which also enables visitors to experience. In this way, the development of micro tourism has been further promoted; (2) enhancement of people's awareness of leisure. In recent years, the purpose of people's tourism has changed from traditional sightseeing to knowledge acquisition, and to leisure vacation experience. They hope to participate in tourism activities in their own way to enjoy the beauty (Feng, 2014). Influenced by change of ideas toward tourism, tourism consumers' behavior has also changed greatly. The tempo has changed from quick to slow, the state has changed from walk to stop, and the action has changed from passive to active. All of these coincide with micro-tourism, so micro-tourism enjoys increasing popularity among the public; (3) the guidance of social policies. First, it is the guidance of national policies. In February 2013, the General Office of the State Council issued The Outline of National Tourism and leisure, which advocated to construct a national tourism and leisure system with Chinese characteristics; in August 2014, China issued Several Opinions of the State Council on Promoting the Reform and Development of the Tourism Industry, which put forward the scientific concept of tourism which focused on the concept of innovative development, and the accelerated transformation of the development (Xiang, 2014); secondly, with the implementation of Notice by the Ministry of Agriculture of the China National Tourism Administration on Vigorously Promoting the Development of Rural Tourism in China, rural tourism has become mature. Based on the perception of the simplicity of rural life, the understanding of rural culture and the longing for idyllic scenery, micro-tourists favor rural areas which are closest to nature. And rural tourism has become a manifestation of micro-tourism. "Micro-tourism", as a new product, has realized the integration and upgrading of the past urban, suburban and rural tourism with its personalized characteristics. And rural tourism is the best destination for micro-tourists to experience rural life, labor and folk customs; finally, with the adjustment of golden holidays, the new vacation system has increased a number of short holidays for short-distance travel. Paid holidays also humanely enable people to choose off-peak and off-season travel. The adjustment of holidays makes people more willing to engage in individual travel flexibly and independently. A series of policies favor the concurrent development of tourism and leisure holidays. The era of "rigid demand of leisure" has arrived in an all-round way, which invisibly promotes the emergence and rapid development of micro-tourism.

\subsubsection{Meaning of "Micro" Concept and Characteristics of Micro-Tourism}

As micro-tourism is a popular vocabulary that emerges in recent years, there is no unified conclusion on its concept. The earliest definition comes from the Xinmin Evening News in 2011: "micro tourism" refers to short travel that one can start at any time. There is no need preparing too much luggage, and spending long time on careful planning and arrangement; one may go to a strange city, take a strange road, see everything that is totally strange, or stay in the city where one lives, escape from electronic products, just read books, enjoy sunshine, and doze off. Or even one can just slowly enjoy a delicious meal or try farm dishes under the shed of loofah (Shen, 2011). Since then, this expression has been widely used. Compared with long-distance travel, the form of micro-tourism is simpler. One may pick up the backpack and go travelling. And the travel time and ways of travelling are more arbitrary. Even for a sudden thought of going out on Friday night, one can make it by finding some strategies on the Internet (Note 2).

In foreign countries, there is no such expression as "micro-tourism". They only have a general idea of leisure 
tourism. So to a certain extent, it is a new form of tourism with Chinese characteristics, as well as a localized exploration. In the foreign research literature, and in a number of databases, the author of this paper uses "Micro-Tourism", literally translated from its Chinese term, as the main content of the retrieval. It is difficult to find relevant literature. Then the author of this paper searches the term on the website and finally finds some relevant documents. In 2002, Neil who came from University of Queensland, Australia became the first to discuss the relationship between leisure and tourism. He made a comparative study on the difference between leisure behavior and tourism experience behavior (Carr, 2002). In China, more and more people in academic circles discussed the concept of micro tourism: Jia Yunfeng (2012) held the view that micro tourism is different from mid-long distance travel that takes long time and covers long distance. It is featured with short time, close distance, low cost and slow experience. The purpose of micro tourism is to avoid the tiring long journey and the cumbersome travel preparation and to enjoy a thorough relaxation at a place nearby on a whim and experience another kind of leisure tourism with the joy of life. Xiong Lirong (2013) and others believed that micro-tourism refers to a travel that takes advantage of rather short leisure time (Xiong, 2013). He Xin and Wang Jingqiang (2014) analyzed the temporary, allopatric and unproductive nature of "micro-tourism", and compared "micro-tourism" with short-distance tourism, believing that "micro-tourism" is a way of leisure with tourism attributes (He \& Wang, 2014). Based on the survey data, Fu Yuanbai (2015) analyzed the micro-tourism market of the citizens in Ningbo, and concluded that micro-tourism is a kind of short-distance leisure and experience tourism activity that uses fragmented leisure time to relax, and to release pressure, avoids redundant planning and preparation and follows one's will (Fu, 2015). Zeng Chao (2016) first clarified the concept of micro-tourism in his research. He believed that micro-tourism is not only manifested in the form of "short", but also in the spiritual connotation featured with "life attitudes", "public welfare" and "personal attributes" (Zeng, 2016).

To sum up, the author of this paper deems that the concept of micro-tourism can be understood as: a kind of leisure and experience tourism that takes less than 2 days and the consumption is low. One may utilize scattered leisure time and freely go to outskirts of the suburb, the quiet rural village or mountain areas without long-term planning and deliberate preparation so as to release the pressure and to sooth the mood. "Micro-tourism" is designed for people to discover the good things around them in the spirit of free exploration, which is distinguished from the short-distance tourism provided by common travel agencies. Its essence is the new concept and new form of in-depth development of urban tourism (Xiong, 2013). Therefore, micro-tourism carries the following typical characteristics:

\section{(1) Subject of Micro-Tourism}

The subject of micro-tourism is a relatively small market, and there is a big difference between micro-tourists and the organized mass tourists (Xiong, 2013). Micro-tourism mainly refers to independent travelling where tourists are the main decision-makers. And individual tourism embodies personalized characteristics of micro-tourism. In modern society, young tourists have diversified individual needs, which may include their desire for adventure, novelty and quietness. Different from original backpacker tourism, tourists utilize modern Internet information, freely make travel arrangements, and freely choose their destinations, of which tourism destinations may be non-scenic spots or non-scenic areas.

\section{(2) Micro Time}

Micro-travel time is basically restricted in one day or two days at most, mainly at weekends (not overlapped with short holidays), or on the leisure time on workdays. It is similar to one-day tour, two-day tour and weekend tour in terms of time. Choosing micro-tourism, one can make full use of these fragmented leisure time, without deliberately breaking the daily plan. Just taking out two days or less, one will have a relaxed and leisure trip to release the pressure of body and mind. Hence, micro-tourism is now a new form of tourism that can meet people's needs.

\section{(3) Micro Space}

As micro-travel time is short, micro-tourism doesn't not like the long-distance travel in space. Compared with the original tourism mode, the destination of micro-tourism is usually chosen close to the place where one sets out, usually in places in the city or its surroundings. One may check the distance and then choose to travel by automobiles, bicycles or even on foot. With the continuous development of modern transportation technology, micro-tourism by cars, motor vehicles or high-speed rails has sprung up rapidly. Usually, the spatial span of micro-tourism activities is within 300 kilometers' range in and around the city. But it is foreseeable that this scope will continue to expand with the advancement of transportation technology in the future. 


\section{(4) Micro Consumption}

Micro-tourism activities are relatively simple, which doesn't require too much input costs and tickets. And the low product pricing is acceptable to ordinary micro-tourism tourists, and may continue to attract their attention or repeated purchases, making the micro-tourism market long-lasting.

\section{(5) Micro Experience}

Nowadays, tourists are no longer satisfied with stereotyped and stop-and-go tourism, but pay more attention to personalized tourism experience. Micro-tourism is a temporary release for tourists to stay away from their daily work, study and living environment. In their travelling, what tourists pursue is a physical and mental experience different from the usual environment and a more personalized tourism experience than other tourist groups, which means micro-tourism should provide a different experience for micro-tourists.

\subsection{Propagation Characteristics in the Internet Age}

From the PC era to the Internet era, the Internet has brought the cultural communication of society into a new era. It shortens the distance between time and space, and the world has been contracted into a new dimension space, where we can browse the customs of all countries in real time with fast, extensive, timely, cheap and interactive dissemination of information. Compared with the traditional media, the Internet has many distinct advantages: (1) real-time information. Micro age makes information transmission highly efficient, removing barriers from the occurrence of the event to its information acquisition and making the "sharing new things anytime and anywhere" easier and faster. (2) live experience. The information of many events is released at the first time. Supplemented by pictures, videos, audios and other effects, they can make the audience feel as if they were on the scene of the event. At the same time, the audience can make interactions by timely feedback their suggestions and opinions through comments. (3) cracking dissemination. In the Internet era, information spreads to people everywhere just like nuclear fission. Mass audiences can get deeply involved in the organization, production and dissemination of information through digitization. Network information sources become rich and scattered instead of merely coming from a single channel. (4) flattening propagation. In the Internet era, digital technology blurs the boundary between communicators and audiences, and communication activities gradually become "decentralized", presenting a network and two-way structure of information transmission, with an obvious flattening trend. At the same time, the Internet era also has certain disadvantages, such as information asymmetry, group "information cocoon" effect (Deng, 2016 \& Yang, 2014), information garbage, and excess of information.

\section{Current Development of Micro-Tourism}

From the product selection of micro-tourism, with the emergence of "micro-tourism", the market products of micro-tourism are being produced one after another. Tourism websites have become an important driving force for the development of micro-tourism. In recent years, self-service travel market, including micro-tourism, has been growing rapidly. The number of self-service routes to the same destination rises over that of the group tour routes. According to the information released by Xinhuanet, during this year's National Day Golden Week, many tourist websites have catered to the emerging micro-tourism market. Combined with market demand, the "micro-tourism" group buying has been launched. Most of the group buying products are based on 1-2 days short distance travels and 1 day hotel accommodation (Diao, 2012). Among numerous online group buying websites, Nuomi.com has set up a tourism group buying channel to launch 117 tourism group buying activities covering Beijing, Shanghai, Hong Kong and other cities. Among these activities, the number of purchase of rooms in 7 days Inn is the most popular, with more than 23,000 people booking rooms on the first day. Meanwhile, it is found from the monitoring data of Tuniu.com that the number of independent tourists in July and August of 2011 increased by three times, compared with the same period of last year, among which "micro-tourism" and travel experience achieved through Weibo interaction continues to grow (Sun, 2011). At the same time, traditional travel agencies have begun to pay attention to the "micro-tourism" market, and have launched corresponding "micro-tourism" packages, turning original fixed travel routes into combined, menu, and optional routes. Tourists can choose self-driving tour in combination with tickets and hotels, or semi-self-service tour by bus or short-distance train with tickets and hotels. During the Spring Festival of 2012, travel website Long369.com specially opened a peripheral tour column, and launched "micro-tourism" products ranging from 1 day to 8 days, covering tourist destinations such as Jinan and Tai 'an, which enjoyed immense popularity in the market (Sun, 2011). 


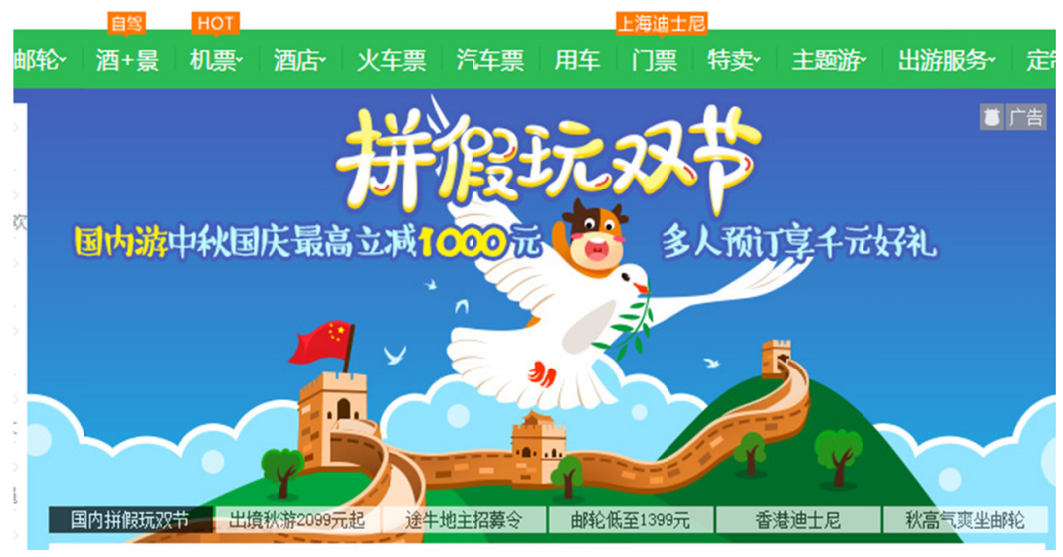

Figure 1. Holiday tourism products launched by Tuniu.com

In terms of the travel time of micro tourism, it is found from Report on Online Booking of Peripheral Self-Service Travels 2014, released by online tourism platform Lvmama, China's leading brand in self-service travel as well as the year's gold medal winner of China self-driving tour's route design, that self-driving tourists mostly spent one day travelling around, usually at weekends, and most of them set out on Saturday. In addition, Lvmama has held several thousand participants' self-driving travels at different destinations and hundreds of hundred participants' group self-driving travels at weekends. There are hundreds of thousands of people surveyed participating in such activities (Note 3). According to the data survey of Analysys, free travel in China carries the characteristics of short time and high frequency; Saturday and Thursday are the peak days for those searching travel information, and weekend trips are becoming major travels for free-riders. It is evident that multiple trips a year have become a rigid demand, and the consumer market is being upgraded.
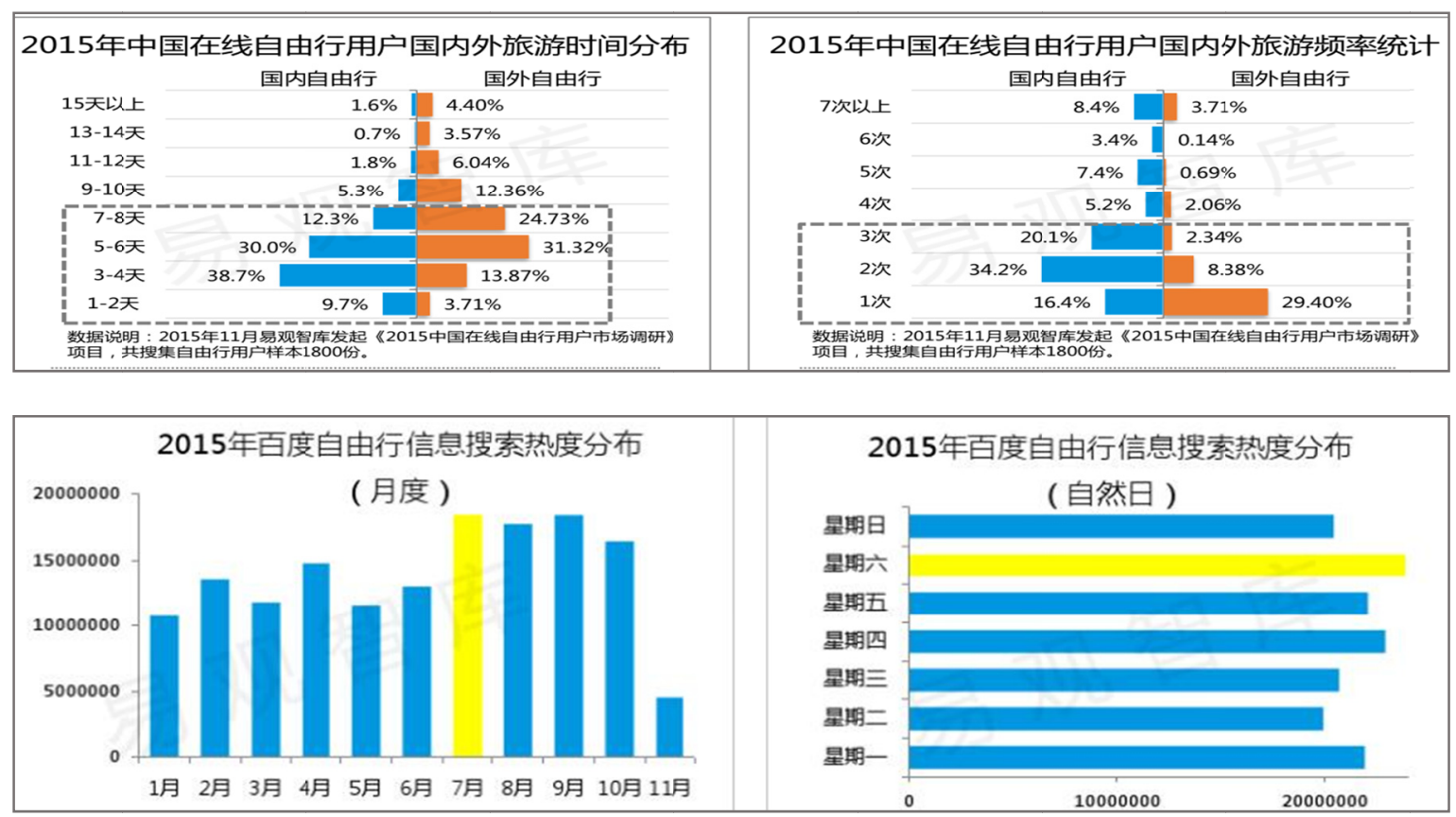

Figure 2. Related statistics on individual tourists' travel frequency and time selection (Note 4)

From the number of tourists and subject characteristics, according to the report released by the Office of the National Holiday Tourism Inter-Ministerial Coordination Meeting: During the golden week of National Holidays in 2013, China received 428 million tourists, of which 334 million tourists chose one-day travel, accounting for 
$78 \%$ of the total number, $1.8 \%$ higher than the number of tourists received during Mid-Autumn festival and the National Day holidays in 2012. During the golden week of Spring Festival, China received 203 million tourists, of which 155 million tourists chose one-day travel, accounting for $76.4 \%$ of the total number. In 2014 , China received 475 million tourists, of which 375 million tourists chose one-day travel million, accounting for $78.9 \%$ of the total number, $12.3 \%$ higher than the number during the National Day holidays in 2013. During the golden week of Spring Festival, China received 231 million tourists nationwide, of which 178 million tourists chose one-day travel, accounting for $77.1 \%$ of the total number (Note 5).

According to China's Online Travel Booking Service Users Report, 2010-2011 released by iResearch Consulting, in 2010 online travel booking users were mainly males with high education and high income, and the average monthly male users accounted for $52.9 \%$; More than half of the users acquired a bachelor's degree, and the average monthly users accounted for 50.2\%; Most of their monthly income reached RMB 1,000-2,000 yuan, with average monthly users accounting for $23.1 \%$; there are also a number of people whose monthly income reached RMB 2,000-3,000 yuan or RMB 3,000-5,000 yuan (Note 6); according to the earlier statistical analysis of data from Analysys think tank, major independent travel users' tourism consumption stood at RMB $1,501-5,000$ yuan, and less than $10 \%$ of these tourists' consumption was below RMB 1,500 yuan; over $40 \%$ of users spent more than RMB 8,000 yuan on their annual travel. The post-80s and post-90s have become the major consumption body of free travel, among which the consumption ability of the post-90s became the age group most willing to spend on free travel, exceeding that of the post-80s (Zeng, 2016).

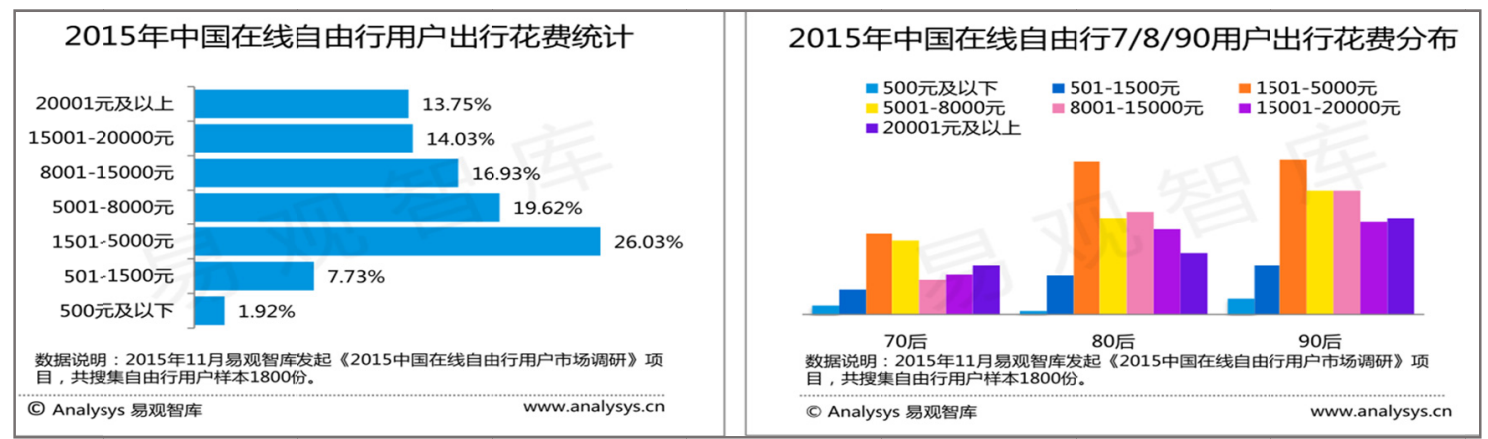

Figure 3. Consumption characteristics of free travel users at different age (Note 7)

From the analysis of the development of micro-tourism market, as an emerging tourism industry, the micro-tourism market in China is very popular at present and is drawing more and more attention. However, at the same time, the construction of related system of micro-tourism is relatively backward. As micro-tourism emerged recently, the construction of micro-tourism destination is still in an early development stage, and personalized micro-tourism products are still far from meeting the growing needs of tourists.

Meanwhile, some micro-tourism products are not favored by micro tourism users, and micro-product suppliers also face some doubts. For instance, the so-called "micro-tourism" products remain traditional tourism products packaged with a novel name. These doubts will have a negative impact on the development of micro-tourism. Therefore, it is urgent to make in-depth and detailed investigations and analyses on various aspects of micro-tourism in China and to design products in line with the needs of the micro-tourism market.

\section{Research on Micro-Tourism Market in Changsha City}

\subsection{Analysis of Micro-Tourism Resources in Changsha City}

In essence, the development of micro-tourism also relies on regular tourist resources. Hence, micro-tourism resources originate from regular tourism resources. In terms of quantity, there are abundant tourism resources in Changsha city. Up to now, Changsha boasts a total of 80 tourist areas, including 1 state-level scenic spot, 1 national 5A grade scenic spot, 14 national 4A grade scenic spots, 5 national forest parks, 26 national key cultural relics protection units, 2 national water parks, 11 provincial-level scenic spots, 4 provincial-level forest parks, 2 cultural heritages ranking top 10 in the province, and 74 provincial-level cultural relics protection units. In fact, due to the randomness and freedom of micro-tourism, scenic spots with potential development of micro-tourism are far beyond the above mentioned scenic areas. Those unrated public parks, leisure spots and other places can also be served as destinations of micro-tourism, such as Kaifu Temple, Changsha Tongguan Kiln Site, Sanshixu 
Site, Former Residence of Jia Yi, Former Residence of Gan Siqi, Former Residence of Tao Shiyue, Tomb of Zuo Zongtang, Tomb of Pei Xiu, Tomb of Guo Liang, Tomb of Yi Pu, Pozi Street, Taiping Street, Shen's Grand House, Jinggang Town, Wenjiashi Town, Langli Town, Hunan First Normal University, Changsha Sea World, Shutang Mountain, Eryang Mountain, Jijia Mountain, Peach Blossom Mountain Park, Wushan Mountain, Moon Island, Liuyang Gufeng Cave, Huangcai Reservoir Water Park, Changsha Section of Xiangjiang River, Laodao River, Jinjiang River, Gushan Forest Park, Daowu Mountain Scenic Area, and Huilong Mountain. Since these scenic areas have not yet been classified in the relevant authoritative statistical yearbook and official report, the author of this paper merely collects and sorts out these data after referring to Google earth and Baidu Map. Attractions in bold front are identified as key scenic spots that enjoys priority development.
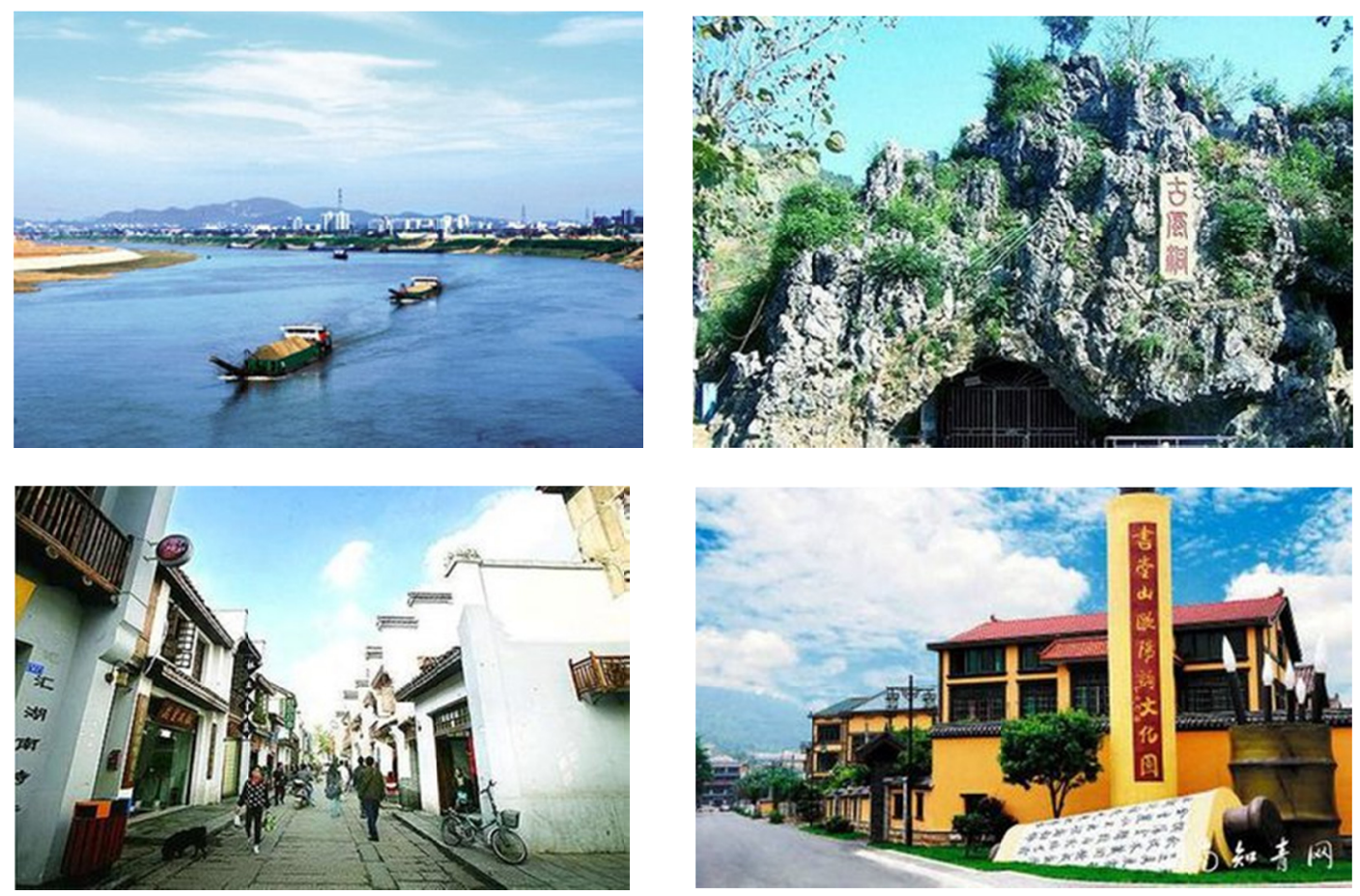

Figure 4. Samples on micro tourism resources in Changsha City

\subsection{Analysis on Comprehensive Environment for the Development of Micro-Tourism in Changsha City}

(1) The natural environment and tourism resources environment are suitable for the development of micro-tourism.

Superior natural environment creates abundant tourism resources and rich historical culture in Changsha, which lays a resource foundation for the initiation and development of micro-tourism. As a whole, it boasts superior geographical location, diverse topographical features, profound history and culture, outstanding human resources, abundant hydrologic resources and excellent climate and biological resources, as well as superior location and traffic conditions. Immense tourism resources here render huge potential with its diverse landscapes, forming unique "Hunan culture" resources with the integration of the regional environment.

(2) The socio-economic environment is conducive to the development of micro-tourism.

The way to measure whether or not a region has development potential of micro-tourism mainly involves the analysis of the overall socio-economic development, the popularity of tourism investment and the ownership of private cars as the main travel tool (Yang, 2015). The overall characteristics may include higher socio-economic levels, rapid development, unreduced tourism investment fever, and high private car ownership.

\subsection{Strategy for the Development of Micro-Tourism in Changsha City Under the Background of the Internet Era}

According to the above analyses, Changsha micro-tourism has already have a good foundation for development, but it still needs to strengthen online and offline interaction to stimulate the micro-tourism market demand. Based on the current tourism development in Changsha, the author of this paper believes that we can start from 
the following aspects:

\section{(1) Follow the "Micro" Principle}

The distance between the activity space of micro-tourism and tourists' departure place is relatively close, so the regional space of micro-tourism should not be too distant. In accordance with the "distance-decay regularity" and the current traffic condition, the distance should be controlled within the range of $300 \mathrm{~km}$. For example, we can develop peripheral tourism and suburban tourism around Changsha city; For farther areas, we can develop 1-day tour, 2-day tour, self-driving tour, etc. When planning micro-tourism projects, we should include unconventional scenic spots such as cities and surrounding towns into micro-tourism scenic spots to provide free access for micro-tourists, which may produce personalized and characteristic landscape effects. Self-service tour, rural tour, agricultural tour, forest tour and fields tour can also be considered. while developing micro-tourism products, we should follow the principle of sustainable development to ensure the synchronization of environmental ecological benefits, social benefits and economic benefits for sustainable development of tourism .

\section{(2) Create "Micro" Products}

With the advent of the era of Internet, micro-tourism has injected new vitality to the quiet tourist market, bringing infinite business opportunities to tourism enterprises. To grab the "micro" business opportunities, enterprises should capture the psychological needs of tourists, make dedicated efforts on product design, and focus on developing quality tourism products, making the public enjoy and love travel. Tourism enterprises also should strive for more innovative ideas, encourage and support staff to innovate, actively investigate and analyze the tourism needs of micro tourists, and design new, unique or special micro tourism products which emphasize experience.

At present, some travel agencies has begun to pay attention to the development of micro tourism market on their website. They innovate the packaging of traditional tourism products, namely, changing fixed traditional tourist routes to menu, combined or optional ones, and launch short-distance "micro-tourism" packages. This kind of package can satisfy tourists' individual requirements. Different from traditional tourist routes, it provides self-service products, which is convenient and simple. Now, it has gradually become a trend for travel agencies to customize micro-tourism.

\section{(3) Carry out "micro" marketing activities}

In today's world where online marketing is popular, to occupy tourism market and to effectively attract tourists, micro tourism enterprises should build diversified interactive advertising platform such as SNS, Weibo, WeChat, micro-movies, ctrip.com, Qyer.com, and Lvping.com. And they should conduct necessary word-of-mouth marketing and presents micro tourism products' advantages, shining points and potential value on the basis of analyzing features of traditional target market and potential market.

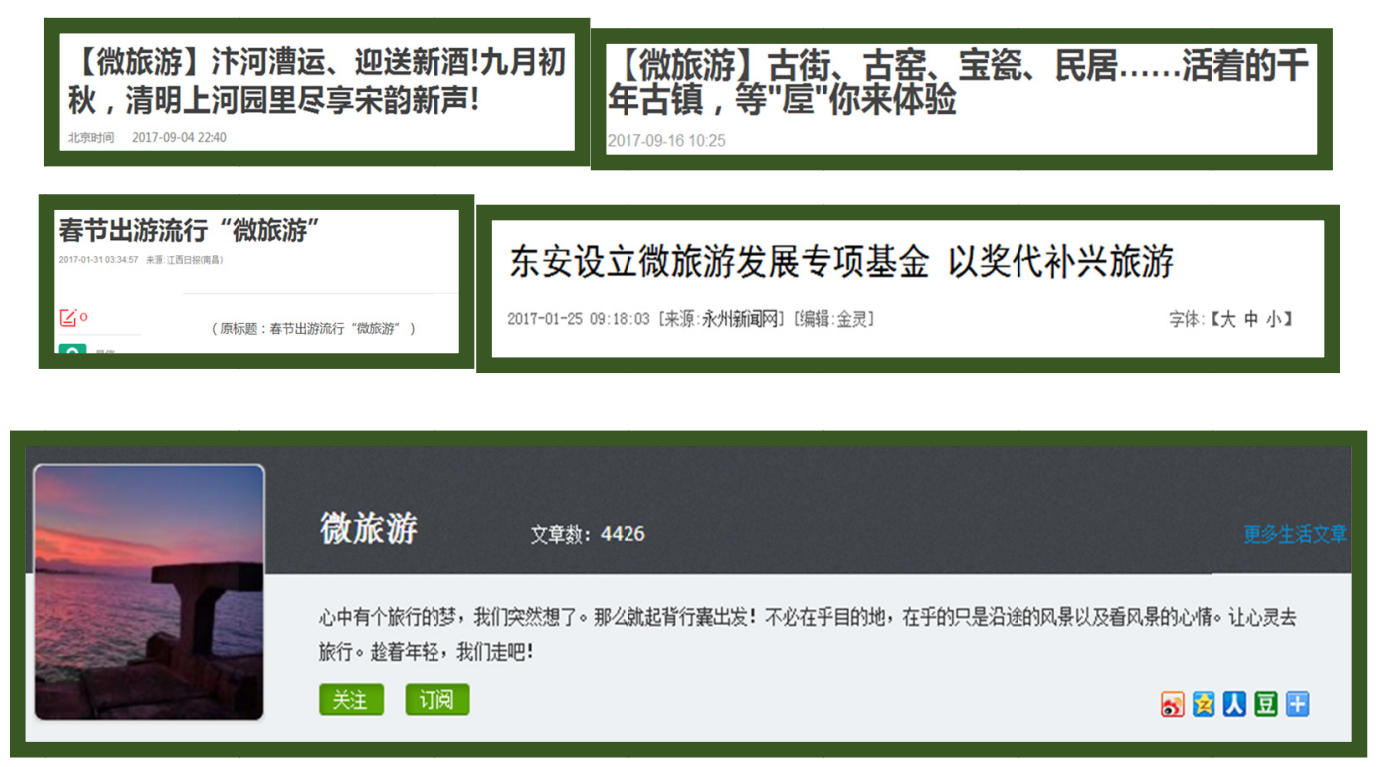

Figure 5. Marketing of micro tourism in scenic spots 


\section{(4) Improve "Micro" Policies}

The development of micro-tourism requires the strong support of tourism departments and related institutions in many aspects, and relevant tourism policies, rules and regulations also need to be formulated and implemented under the leadership of government departments. With the continuous development of micro-tourism in various places, we should work to improve tourism environment. Currently, there is no complete management system and policy on tourism. Therefore, we should establish a cross-departmental organization in charge of coordination and management on the reform and innovation of financial policy, reform of interest distribution mechanism, micro tourism education policy, and micro-tourism safety ensuring system.

All in all, the exploitation and development of micro-tourism needs the government to guide, tourism enterprises to assist, and related departments to fully play their functional role. They should contribute joint efforts to continuously innovate micro-tourism products, improve tourism service quality, strengthen the exploitation of tourism products which emphasize leisure experience, and to think what tourists might think. In doing so, they can thus gain benefits from the development of micro-tourism, guide Changsha city's micro-tourism with better results, and adapt to the transformation of tourism industry. As micro-tourism environment is increasingly mature, the Changsha city's micro-tourism is bound to become more rational and mature.

\section{References}

Carr, N. (2002). The Tourism-Leisure Behavioral Continuum. Annals of Tourism Research, (4), 970-985. https://doi.org/10.1016/S0160-7383(02)00002-6

Deng, Q. (2016). A Study on "Information Cocoon" Phenomenon of New Media. Radio \& TV Journal, (8), 96-97.

Diao, L. F. (2012). Business Opportunities in Micro Tourism: Short Journey, Low Cost, Distinct Features. China Tourism, February 27.

Feng, Y. Y. (2014). The Review of National Leisure Research in Recent Ten Years. Journal of Hubei Polytechnic University, (2), 35-40.

Fu, Y. B. (2015). Demand Analysis of and Strategies for Micro-tourism of Ningbo. Journal of Ningbo University (Liberal Arts Edition), 28(3), 91-95.

He, X., \& Wang, J. Q. (2014). Micro Travel: Connotation, Reason and Feature. Journal of Hubei University of Arts and Sciences, 35(2), 70-73.

Jia, Y. F. (2012). Tourism in Micro Age Needs "Micro Planning". China Tourism, February 13.

Shen, M. L. (2011). Enjoying Sunshine not Far Away, Urban White-Collars Choose Weekend "Micro-Tourism". Xinmin Evening News, May 2.

Sun, W. L. (2011). Popular "Micro-Tourism": Pick up Your bag and Go. Xinhua Daily Telegraph, October 6.

Sun, Y. (2013). New mode of Tourism Marketing in the Micro Era - Weibo marketing of Tourism. News, (1), 87-89.

Xiang, Y. S. (2014). Spring of Tourism Industry - Interpretation of Several Opinions of the State Council on Promoting the Reform and Development of the Tourism Industry. Tourism Times, (10), 6-7.

Xiong, L. R., \& Zhang, W. J. (2013). A Study on New Forms of Urban Tourism from the Rise of "Micro-Tourism". China Tourism, August 23.

Yang, H. (2014). Research on the "information cocoons" effect of Microblog. Hunan Normal University.

Yang, S. S. (2015). Empirical Study on Micro-Tourism Product Proliferation Based on RMP Theory - A Case Study of Chengdu. Lanzhou University.

Zeng, C. (2016). The Concept, Status and Trend of Micro Travel. Jiangsu Commercial Forum, (1), 61-73.

\section{Notes}

Note 1. SINA.com. Ministry of Industry and Information Technology: China's mobile phone users reached 1.306 billion in 2015. [EB/OL].http:tech.sina.com.cn/t/2016-01-28/docifxnzanh0236167.shtml,2016-01-28.

Note 2. Sun Weili, Pick up the Backpack and Go - Why "Micro-Tourism" Becomes a New Favorite During Holidays? 
http://news.ifeng.com/gundong/detail_2011_10/05/9653083_0.shtml. October 5, 2011.

Note 3. Lvmama. Report on Online Booking of Peripheral Self-Service Travels 2014. [EB/OL].http://www.Ivmama.com/infolhytuijian/2015-0305-252587.html,2014-01-01/2014-12-31.

Note 4. Data source description: In 2015, analysys launched the project of Research of the Market of China's Online Free Travel Users, and collected a total of 1800 samples of free travel users.

Note 5. Data comes from relevant reports released by the Office of the National Holiday Tourism Inter-Ministerial Coordination Meeting and the official website of the National Tourism Administration.

Note 6. Data comes from iResearch Consulting. Online travel booking service users are males with higher $\begin{array}{llll}\text { education and income. } & \text { [EB/OL]. }\end{array}$ [2012-03-30].http://ec.iresearch.cn/55/20110830/148680.shtml.

Note 7. Data source description: In 2015, analysys launched the project of Research of the Market of China's Online Free Travel Users, and collected a total of 1800 samples of free travel users.

\section{Copyrights}

Copyright for this article is retained by the author(s), with first publication rights granted to the journal.

This is an open-access article distributed under the terms and conditions of the Creative Commons Attribution license (http://creativecommons.org/licenses/by/4.0/). 